\title{
Osteochondritis dissecans of the talus
}

\author{
GIACOMO ZANON ${ }^{1}$, GIOVANNI DI VICO², MATTEO MARULLO ${ }^{1}$ \\ ${ }^{1}$ Department of Orthopaedics and Traumatology, University of Pavia, IRCCS Fondazione Policlinico San Matteo, \\ Pavia, Italy \\ ${ }^{2}$ Casa di Cura S. Michele, Maddaloni, Caserta, Italy
}

\begin{abstract}
Osteochondritis dissecans (OCD) is an acquired idiopathic lesion of subchondral bone that can produce delamination and sequestration with or without articular cartilage involvement and instability.

The cause of OCD is still debated: the most recognized etiology is the occurrence of repetitive micro-traumas associated with vascular impairment, causing progressive ankle pain and dysfunction in skeletally immature and young adult patients.

Ankle OCD is classically located in the medial part of the talus, while lateral and posterior involvement is less frequent.

Diagnosis of OCD, based on MRI findings, is quite straightforward; MRI examination can also be very useful for dating the defect and obtaining information about the associated bone bruise.

Osteochondritis dissecans, if not recognized and treated appropriately, may lead to secondary osteoarthritis with pain and functional limitation.

Surgical treatment is mandatory especially in young patients with unstable cartilage fragments. There are various surgical options: fixation, microfracture, or substitution using autologous chondrocyte implantation techniques.
\end{abstract}

Key words: ankle, autologous chondrocyte implantation, microfracture, osteochondritis dissecans, talus.

Corresponding Author:

Giacomo Zanon, MD

IRCCS Fondazione Policlinico San Matteo, Pavia, Italy

Phone: +39 3404939834

E-mail: zanon.g@libero.it

\section{Introduction}

Osteochondritis dissecans (OCD) of the talus is a subchondral bone pathology that presents as an osteochondral lesion of the talar dome with consequent articular cartilage abnormalities. In 1888 Konig was the first author to use the term osteochondritis dissecans to describe loose bodies found in the knee joint; he believed them to be fragments from an avascular bone lesion (1). In 1922 Kappis was the first to describe a similar lesion in the ankle joint (2). The talus is the third most frequently affected anatomical site, after the knee and the elbow joints $(3,4)$. According to the literature, OCD of the talus has an incidence of $0.09 \%$ and a prevalence of $0.002 / 100,000$ person/year $(5,6)$. OCD is most frequent in the second decade of life, even though its clinical manifestations can appear later than this (7).

Before taking an in-depth look at this disease, it is important to point out that it is the subject of considerable semantic inaccuracy, perpetuated since 1959 when Berndt and Harty developed the first classification of osteochondral lesions of the talus (8). These authors, assuming the disease to have a traumatic etiology, united under the same name two lesions that other authors subsequently considered, from different perspectives (etiology, epidemiology, clinical presentation and treatment), as separate entities. In addition, subsequent histological studies failed to confirm an underlying inflammatory basis for this disease. Therefore, although the term "osteochondritis" is commonly used in clinical practice, it would be more appropriate to talk of "osteochondrosis", as already suggested by Howald (9).

In the present article, the term osteochondritis dissecans (OCD) will be used to refer to mainly non-trau- 
matic lesions in the child or adolescent, while the term osteochondral fractures will instead be used to indicate injuries of traumatic origin involving both the cartilage and the subchondral bone and occurring predominantly in adults.

\section{Etiology}

Although OCD of the talus is, by definition, detachment of an osteochondral fragment of the talar dome occurring in a growing patient, symptomatic OCD is more typically observed in adults. This apparently greater frequency of OCD in adults compared with adolescents is explained by the fact that the disease often remains asymptomatic for a long time before finally manifesting itself, after a trauma, in adulthood (10-13).

The etiology of OCD is not yet clearly understood, mainly because histological studies can only be performed on fragments that have already become detached, and therefore in the last stage of the disease. There are various etiological theories for OCD: traumatic, ischemic and micro-traumatic.

Most OCD patients do not have a history of previous traumatic events, and it has been suggested that repetitive micro-traumas could instead contribute to the development of the lesion $(10,13,14)$. This latter hypothesis is supported by the fact that the majority of patients with OCD had long practiced sport at intense levels (15). It is also possible that OCD is more likely to become symptomatic, and thus more likely to be diagnosed, in more active individuals.

On the basis of the most recent histological studies in animals, the ischemic theory seems to be the most likely one (16). During endochondral ossification, the process responsible for the growth of long bones, the epiphyseal cartilage is supplied with nutrients via vessels that arise from the perichondrium and run in channels called cartilage canals (17). As cartilage tissue matures, these channels gradually close and the medullary cavity takes over as the source of nourishment. This "handover" is characterized by the formation of anastomoses between vessels in the epiphyseal cartilage and vessels in the advancing ossification front; however, these anastomoses can be particularly unstable and fail, mainly due to local factors such as lack of chemical or mechanical support for neoangiogenesis (18). The result, in such cases, is lack of vascularization and nourishment in a well-defined area of subchondral bone, which therefore evolves towards avascular necrosis $(19,20)$. This phenomenon can manifest itself either as an area of subchondral bone necrosis with an intact overlying layer of cartilage, a situation showing potential for spontaneous healing, or as an already detached osteochondral fragment. Histological studies of osteochondral fragments removed from adolescents with OCD seem to confirm the above-described pathogenic mechanism.

The complete absence of a calcified cartilage layer and subchondral bone plate in adolescents with OCD is a finding which implies that juvenile OCD develops while the endochondral ossification process is still ongoing; therefore, it is unlikely to be a primary disease of the subchondral bone $(21,22)$. Many genetic risk factors have been proposed. Since the first descriptions of OCD, there have been several reports of families in which two or more individuals were affected (23-25). Rare cases of families with a high incidence of OCD across multiple generations, even in different joints, seem to point to an autosomal dominant form of OCD with variable penetrance $(26,27)$.

Sporadic OCD, which is the most common form, seems to be polygenic in nature; that is, the combined effects of several gene variants determine the risk of latent disease. At present, however, there are no ongoing studies to identify such genes.

\section{Clinical presentation}

As indicated above, the disease often remains asymptomatic for a long time, before being discovered by chance when the patient, even in adulthood, undergoes an X-ray of the ankle for other reasons. When it is symptomatic, the patient complains of intermittent weight-bearing pain, mainly when running.

When the osteochondral fragment is detached, the symptoms are more marked, consisting of: intense pain (the so-called "articular crisis"), joint swelling, instability during walking and even locking. In $6.5 \%$ of cases the onset of symptoms follows an ankle sprain (28). 
The patient should be questioned about the onset of the symptoms, any prior ankle trauma, and the evolution and exact features of the pain. Onset of symptoms immediately after a severe injury of the ankle suggests an osteochondral fracture or (only after excluding this first possibility) post-traumatic displacement of an OCD fragment.

An osteochondral fracture is always found to be the consequence of a serious ankle injury, frequently a sprain sustained with the foot in inversion and internal rotation. Consequently, the most common site of injury is the lateral margin of the talar dome.

During the clinical examination of the ankle, it is important to look for any localized tenderness; the talar dome can be palpated by placing the ankle in extreme dorsal flexion. The range of motion and possible associated ligamentous laxity should be measured, also in comparison with the contralateral side.

\section{Imaging}

The first investigation to perform is the standard radiographic examination. In antero-posterior projection it is often possible to see a subchondral halo, a sign outlining the osteochondral fragment; it is also possible to discern OCD from an intraosseous mucoid cyst or a dystrophic lesion. A radiograph with the ankle in $15^{\circ}$ of internal rotation is particularly useful for investigating the supero-lateral corner of the talus given that, in this position, it is free from fibular overlap. Stress X-rays are not of paramount importance in this disease and may actually be impossible to perform in acute situations.

Osteochondritis dissecans is predominantly located in the supero-medial corner of the talus; it occurs more four times frequently in this site than in the superolateral corner $(29,30)$. The location of the lesion is a factor that differentiates OCD from traumatic osteochondral fracture, which is more frequent on the lateral side. In addition, traumatic injuries are often more superficial while OCD always involves the subchondral bone $(31,32)$. A CT scan without contrast gives precise information about the location and extent of the lesion, but does not allow assessment of the integrity of the cartilage. This can instead be obtained using magnetic resonance imaging (MRI) or CT-arthrography (CTA). Although MRI has the advantage of being non-invasive, it does not allow precise evaluation of the depth of the osteochondral lesion whose edges are blurred by edema of the surrounding bone. Osteochondral fragments appear hypointense on T1weighted images; on T2-weighted images they have a very variable intensity, however they are always characterized by a hyperintense line at their base, which is a sign of the detachment (33) (Fig. 1).
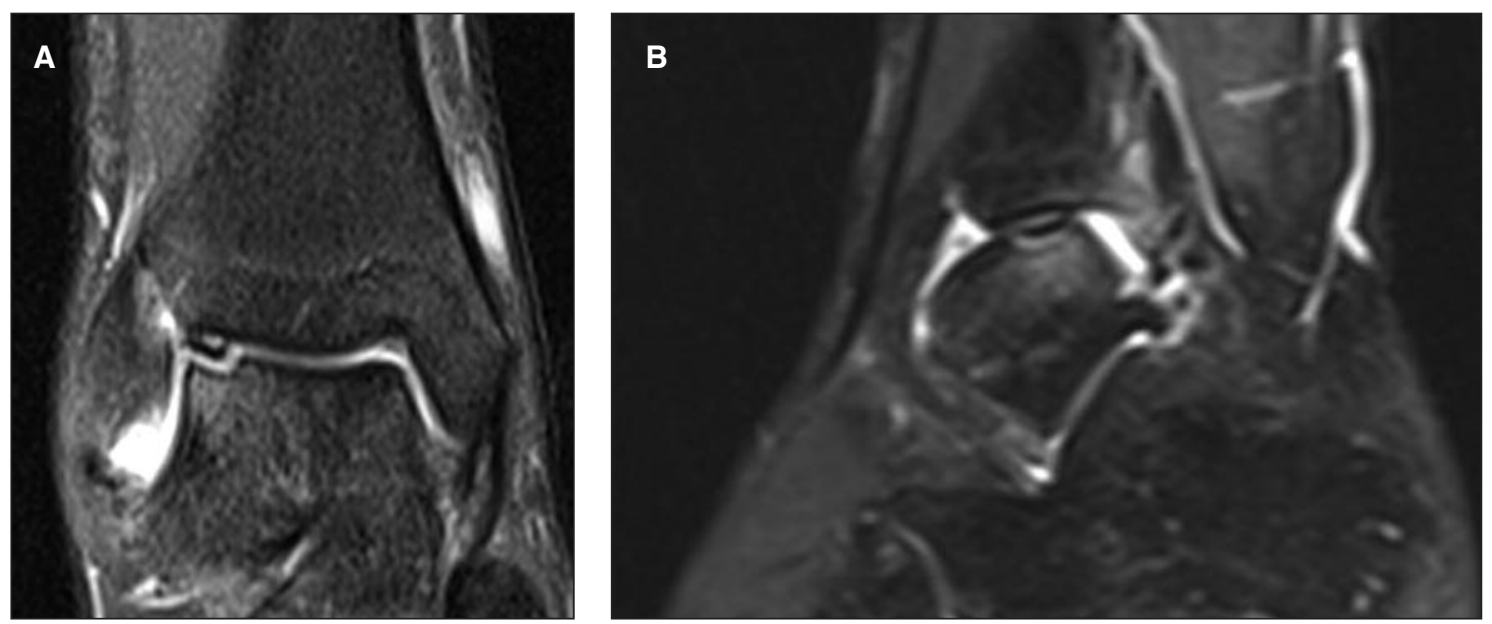

Fig. 1. Magnetic resonance images of the right ankle of a 19-year-old man with symptomatic OCD. A: Coronal proton densityweighted image that shows the OCD lesion detached from the supero-lateral corner of the talar dome, with loss of cartilage continuity. The lesion is classified as stage III. B: Sagittal T1-weighted image showing extensive subchondral bone edema. 


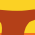
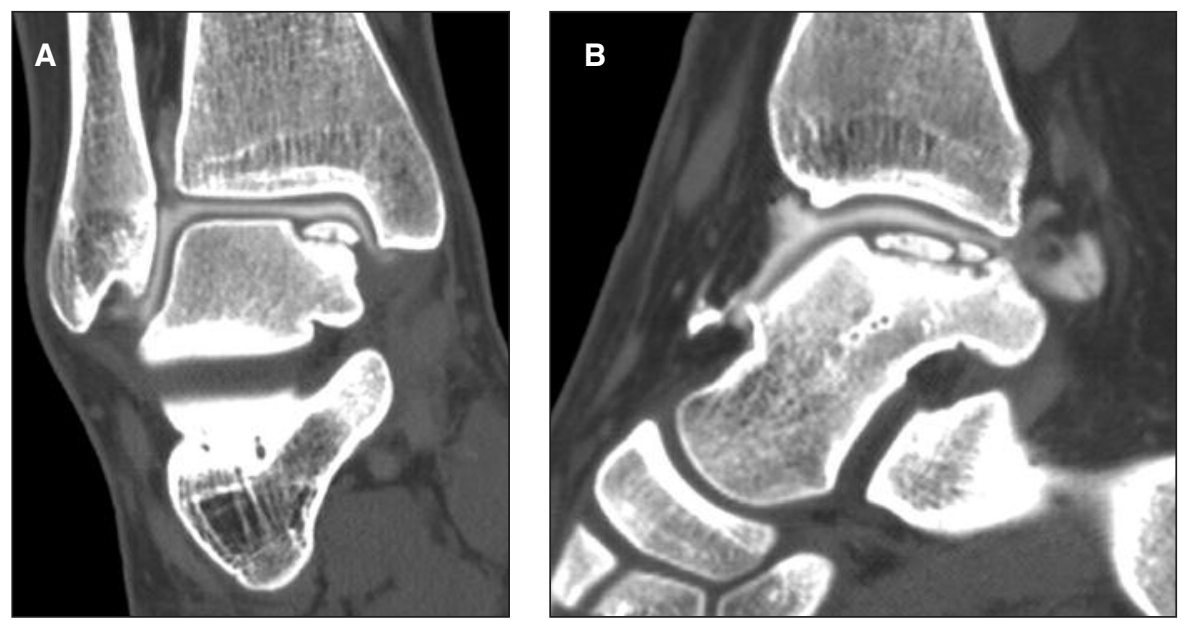

Fig. 2. Arthro-CT images of the right ankle of a 17-year-old girl. The patient is sedentary and asymptomatic. A: Coronal image showing a stage II lesion of the superomedial corner of the talar dome. The contrast medium does not breach the cavity, a sign of integrity of the cartilaginous layer. B: Sagittal image showing the extent of the lesion $(1.7 \mathrm{~cm}$ wide).

In theory, CTA is the examination of choice because it allows effective assessment of both the cartilage and the actual size of the lesion (Fig. 2). However, because of its invasiveness - it requires intra-articular injection of contrast medium and it exposes the patient to ionizing radiation - its use must be carefully evaluated, especially in adolescents.

Although, over the years, various classifications have been proposed using CT, MRI or arthroscopy, Berndt and Harty's classification is still the most widely used (Tab. 1).

Table 1. Berndt and Harty classification (8).

Stage 1. Small area of compression of the subchondral bone

Stage 2. Partially detached osteochondral fragment (flap)

Stage 3. Completely detached osteochondral fragment but undisplaced

Stage 4. Free osteochondral fragment

\section{Treatment}

Treatment of OCD is guided by the patient's age at the onset of symptoms, their severity, and the disease stage according to the Berndt and Harty classification. OCD can remain asymptomatic throughout a patient's life, or it can become symptomatic as a result of a trauma, even in adulthood.

In most cases discovered during adolescence, the overl- ying cartilage layer is completely intact (stage I). Stage I and stage II lesions in teenagers are usually treated conservatively $(31,34,35)$. For stage I lesions, treatment consists of leg cast immobilization and protected weight bearing on the affected ankle for six weeks; physical activity should be limited for at least six months. In stage II lesions, weight bearing is prohibited for six weeks; this period is followed by two weeks of protected weight bearing.

Indications for surgery are: stage III or IV lesion, failure of conservative treatment, detachment of the fragment or decreased potential for revascularization. Younger patients show greater potential for healing with conservative treatment; therefore, while early surgery is indicated in adolescents and in adults, in children younger than 12 years old it is correct to persevere with conservative treatment for up to a year $(5,7,29)$. Pettine and Morrey (35) suggested that surgical treatment should be performed earlier in lesions of the lateral side of the dome. Higuera et al. (30) recommended surgical treatment only for displaced lesions or if revascularization is unlikely. In their series of 18 patients with a mean age of 12.6 years, 13 (73\%) had stage III lesions and 11 underwent conservative treatment. They obtained good to excellent clinical results in $94.8 \%$ of cases, and good to excellent radiographic results in $68.5 \%$. This discordance between clinical and radiographic results in juvenile OCD is found in all the studies. Perumal et al. (36) examined the evolution of 32 OCD lesions treated conservatively in patients with a mean age of 11.9 years; $72 \%$ were stage II lesions. Only $16 \%$ of the patients obtained clinical and radiographic healing after six months of treatment; $77 \%$ of patients were slightly symptomatic at six months with a lesion still visible on plain radiographs; $42 \%$ of them (10 patients) underwent surgery, but 46\% (11 patients) continued conservative treatment for a further six months: all obtained clinical healing, and four also radiographic healing. 
Conservative treatment in the adult population does not give such a high percentage of good clinical results. In a series of 224 grade III and IV lesions, Berndt and Harty (8) documented poor results in $73.9 \%$ of cases. Instead, of the group that underwent surgery (56 patients), 78.6\% had good clinical results.

A more recent systematic review performed by Verhagen et al. (37) reported successful results of conservative treatment in only $45 \%$ of adult patients.

Surgical treatment options include: debridement of the necrotic subchondral bone, internal fixation of the fragment or its removal followed by debridement of the crater, bone marrow stimulation techniques such as drilling or microfractures performed arthroscopically, or tissue transplantation techniques: osteochondral auto or allograft, autologous chondrocyte implantation (ACI), and matrix-induced autologous chondrocyte implantation (MACI). The general principle of surgical treatment is to recreate the cartilage or to refill the defect, restore the articular surface and prevent the evolution towards osteoarthritis.

Zengerink et al. (38) performed a review of the literature on the surgical treatment of OCD lesions. Fragment removal led to a clinical success rate of 54\%; the addition of debridement of the crater increased the success rate to $88 \%$. The association of removal, debridement and marrow stimulation techniques led to the best result, with a success rate of $85 \%$ in stage III or IV lesions smaller than $1.5 \mathrm{~cm}$.

Retrograde drilling is generally indicated in cases of OCD with an intact overlying cartilaginous layer, even if a subchondral cyst is present. The aim of this kind of surgery is to provide blood supply to the lesion without damaging the cartilage. Drilling is performed under arthroscopic control, through the sinus tarsi for medial lesions, or anteromedially for lateral lesions. Kono et al. (39) and Taranow et al. (40) reported success rate of $81-100 \%$.

Transmalleolar drilling should be considered in lesions located in a posterior position in the talar dome, which are difficult to reach. With this technique, a Kwire is drilled into the tibia $3 \mathrm{~cm}$ proximally to the tip of the medial malleolus and directed towards the lesion through the malleolus and the talar cartilage. Robinson et al. (41) described this technique and reported a success rate of $63 \%$.
Arthroscopic microdrilling or microfractures are performed with the aim of breaking the subchondral calcified layer in order to obtain growth factors from the bone marrow; these molecules should induce filling of the cartilage defect with the less specialized fibrocartilage (Fig. 3). Even though their effectiveness in restoring a functional cartilaginous layer is debatable, their value in reducing symptoms has been demonstrated (42). The lesion most suitable for treatment with microfractures is a small $(<6 \mathrm{~mm})$, mostly chondral lesion with little involvement of the subchondral bone (43). Chuckpaiwong et al. (44), reviewing 105 OCD lesions treated with microfractures, reported no failures in the case of lesions smaller than $15 \mathrm{~mm}$ (73 patients), but obtained only one good result in lesions greater than $15 \mathrm{~mm}$ (32 cases) (45). An older age, a high body mass index, a traumatic etiology and the presence of osteophytes are all factors that negatively affect the result.

Arthroscopic debridement, bone marrow stimulation techniques and retrograde or anterograde drilling are the only surgical procedures that can be performed in children and adolescents. Highly invasive techniques such as osteochondral transplant or ACI are not recommended in growing subjects. Moreover, some

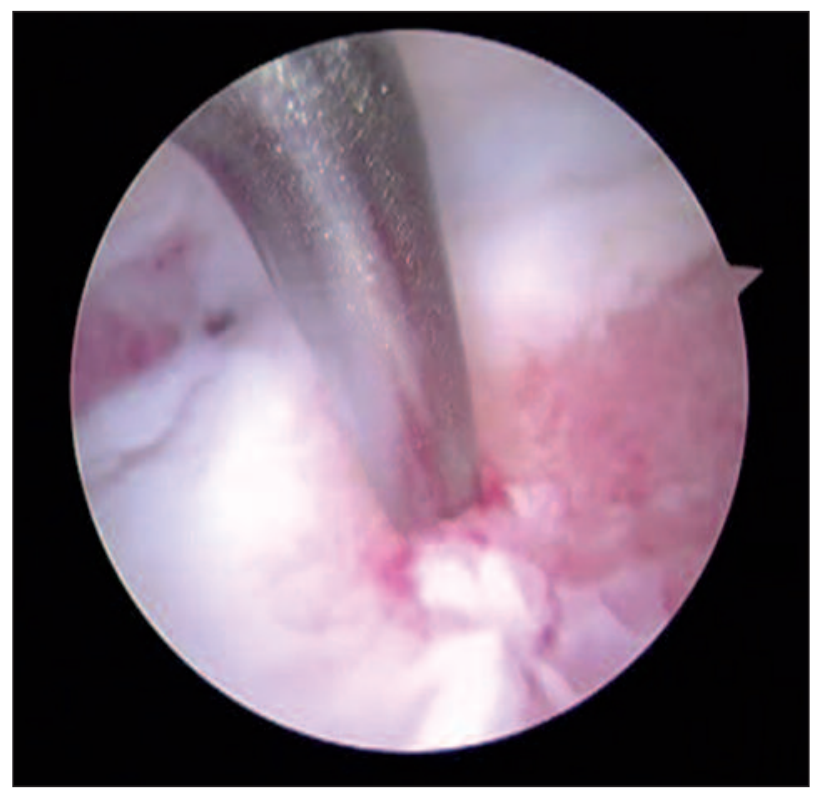

Fig. 3. Arthroscopic image of the right ankle of a 24-year-old footballer affected by stage IV OCD. After removal of the loose body, which was too fragmented to be refixed, microfractures were performed. 
surgical approaches, needing a medial or lateral malleolar osteotomy to reach certain lesion sites, are not feasible because of the risk of growth plate damage and subsequent deviation of the limb axis (Fig. 4).

Fragment refixation is an option to consider in the presence of large loose osteochondral fragments in the joint; however, the subchondral bone should still be vital. Fixation should be performed with metallic screw concealed in the cartilage layer, K-wires, resorbable pins or fibrin glue. This technique has shown a high success rate in recent osteochondral fractures; in chronic OCD lesions the quality of fixation that can be obtained is debatable on account of sclerotic fragment margins.

Several authors have suggested using autografts to treat large stage III or IV OCD lesions $(>1.5 \mathrm{~cm})$. Kouvalchouk et al. (45) proposed a technique consisting of debridement of the lesion and filling of the defect with autologous cancellous bone harvested

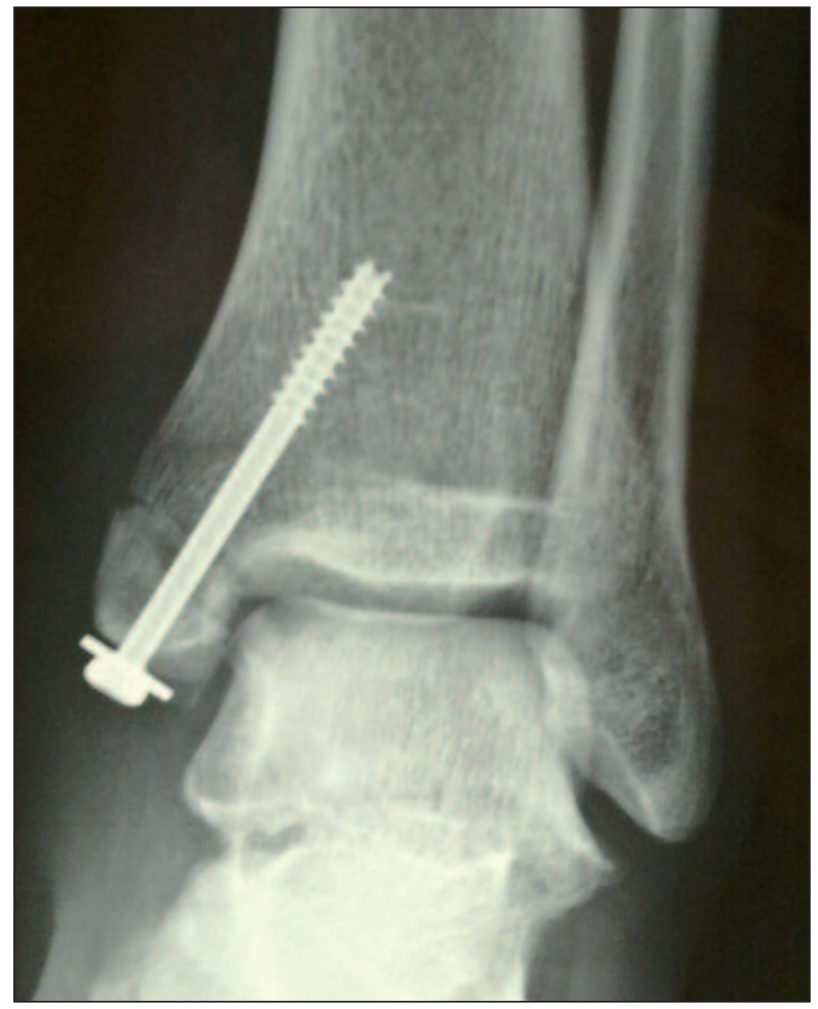

Fig. 4. Postoperative X-ray of a 26-year-old man affected by stage IV OCD located in the postero-medial corner of the left talar dome. The patient underwent autologous matrix-induced chondrogenesis (AMIC). To reach the site of the lesion, a medial malleolus osteotomy was performed and then fixed with a lag screw. from the distal tibia; they obtained good functional results in 22 of 27 patients at one year of follow-up. For extensive OCD lesions, different osteochondral autograft transplantation techniques have been proposed, i.e. the use of a single block graft (osteochondral autograft transplantation, OAT) or several smaller osteochondral blocks (mosaicplasty), and more recently ACI, even associated with a synthetic matrix (MACI). The single block graft is generally harvested from the medial side of the trochlea of the ipsilateral knee; it is then press-fitted in the talar defect. Lesions typically treated with this technique are usually extensive, located on the medial side of the talus and have an underlying subchondral cyst.

The mosaicplasty technique consists of harvesting several small osteochondral cylinders $(3.5-4.5 \mathrm{~mm}$ in diameter) from the non-weight-bearing segment of the lateral or medial femoral ridge of the ipsilateral knee or, if the lesion is smaller, from the most anterior surface of the talar dome. The cylinders are then inserted perpendicularly in the defect. This technique can be used to fill lesions measuring up to $4 \mathrm{~cm}^{2}$.

Techniques used for grafting and restoration of the articular surface have given excellent results. In a series of 50 patients submitted to mosaicplasty, Scranton et al. (46) reported good results in $90 \%$ of cases at a mean 36-month follow-up; Hangody and Fules (47) obtained good or excellent results in $94 \%$ of 36 patients at a mean 4.2-year follow-up. The drawback of this technique is donor site morbidity, which can occur in up to $15-16 \%$ of cases (37).

The ACI technique used for the talar dome does not differ from the more widespread one used for the knee. It consists of two surgical steps. The first one is the harvesting of chondrocytes from the knee or the ankle, arthroscopically performed. The second step is performed after the cells have been allowed to grow and expand in vitro for 6-8 weeks; it consists of an open procedure in which the defect is filled with the cells and then closed with a periosteal patch harvested from the distal tibia.

Giannini et al. (48) reported a series of 10 patients with a mean age of 25.8 years and a mean talar defect of $3.1 \mathrm{~cm}^{2}$, followed up for 10 years after ACI. At the last follow-up, their mean American Orthopaedic Foot and Ankle Society (AOFAS) score was still 92.7 and 
Table 2. Outcome of the various surgical treatment for OCD.

\begin{tabular}{llllll}
\hline Surgical treatment & Number of studies & Number of patients & $\begin{array}{l}\text { Number of patients Success rate in \% } \\
\text { successfully treated }\end{array}$ & $\begin{array}{l}\text { Success range } \\
\text { in \% }\end{array}$ \\
\hline Debridement & 14 & 251 & 177 & 71 & $30-100$ \\
Refixation & 7 & 78 & 64 & 82 & $0-100$ \\
Bone marrow stimulation & 24 & 422 & 319 & 76 & $33-100$ \\
Transplantation (ACI e OATS) & 21 & 352 & 294 & 84 & $1-100$
\end{tabular}

MR T2-mapping findings were comparable to those of native cartilage.

After several studies in the knee joint, the technique of autologous matrix-induced chondrogenesis (AMIC) was also proposed for the talar dome. This technique consists of filling the cartilage defect with a synthetic membrane, usually collagen-based. Its microarchitecture encourages chondrocytes coming from the healthy periphery and of the lesion to grow and to produce extracellular matrix. The AMIC technique replaces only the cartilaginous layer, therefore it must be associated with a cancellous bone autograft to fill the subchondral defect. Valderrabano et al. (49) recently presented a series of 26 patients treated with this technique and followed up for a mean of 31 months.

The clinical results were very good and the mean AOFAS score at the last follow up was 89. Complete filling of the cartilaginous defect was achieved in 35\% of cases; hypertrophic growth was found in 50\%. Seventy-three $\%$ practiced sports before the onset of symptoms; $62 \%$ resumed their sport activity after surgery.

\section{Discussion}

Even through the various techniques proposed for treating OCD of the talus have shown good and promising results, clear indications remain to be developed. Most articles are case series, with a level IV of evidence; they examined small populations and often presented results at short-term follow-up (50). There are no randomized controlled trials in the literature, except for one which involved 15 patients followed up for six months. Moreover, there is no uniformity in cli- nical evaluation, therefore studies cannot easily be compared. Few studies have added instrumental (MRI) or histological findings.

In view of these limitations, surgical treatments for OCD of the talus present an overall success rate of $79 \%$. This rate depends on the stage of the lesion, being lower for stage IV lesions (76\%) and higher for low-grade lesions (82\% for stage I, $86 \%$ for stage II and $83 \%$ for stage III). This finding seems to confirm the paramount importance of early surgical treatment for OCD.

Most patients with stage I-III lesions are treated with bone marrow stimulation techniques; instead the ACI technique and osteochondral autografts are applied in stage IV lesions.

Analyzing the results according to the surgical technique used, ACI and autografts showed a higher success rate $(84 \%)$ compared with refixation of the fragment (82\%), bone marrow stimulation techniques (76\%) and debridement (71\%) (Tab. 2). This finding is particularly interesting because the techniques that showed a lower success rate are the ones usually used to treat early-stage lesions; instead ACI and osteochondral autograft transplantation techniques are commonly used in stage IV lesions.

In conclusion, although OCD of the talus is a confusing disease, also as regards the terminology used, there is a growing awareness of its pathogenesis and evolution. Accordingly, the therapeutic strategies and surgical interventions being proposed to treat it are increasing and improving.

Nevertheless, the results of research in this area are sporadic and, despite a number of promising future directions for the treatment of OCD, more scientific evidence is needed to obtain a treatment that demonstrates adequate safety and efficacy. 


\section{References}

1. Konig A. Uber freie Körper in den Gelenken. Dtsch Z Chir. 1888;27:90-103.

2. Kappis M. Weitese Beitrage zur Traumatisch-mechanischenEntstehung der "Spontanen" Knospelablosungen. Dtsch Z Chir. 1922;171:13-29.

3. Aichroth P. Osteochondritis dissecans of the knee. A clinical survey. J Bone Joint Surg Br. 1971;53:440-447.

4. Steinhagen J, Niggemeyer O, Bruns J. Etiology and pathogenesis of osteochondrosis dissecans tali. Orthopade. 2001;30:20-27.

5. Bauer M, Jonsson K, Lindén B. Osteochondritis dissecans of the ankle. A 20-year follow-up study. J Bone Joint Surg Br. 1987;69:93-96.

6. Flick AB, Gould N. Osteochondritis dissecans of the talus (transchondral fractures of the talus): review of the literature and new surgical approach for medial dome lesions. Foot Ankle. 1985;5:165-185.

7. Bruns J, Rosenbach B. Osteochondrosis dissecans of the talus. Comparison of results of surgical treatment in adolescents and adults. Arch Orthop Trauma Surg. 1992;112:23-27.

8. Berndt A, Harty M. Transchondral fractures (osteochondritis dissecans) of the talus. J Bone Joint Surg Am. 1959;41:9881020.

9. Howald H. Zur Kenntnis der osteochondrosis dissecans (osteochondrotos dissecans). Archiv für orthopädische und Unfall-Chirurgie. 1942;41:730-788.

10. McCullough CJ, Venugopal V. Osteochondritis dissecans of the talus: the natural history. Clin Orthop Relat Res. 1979; 144:264-268.

11. Kumai T, Takakura Y, Higashiyama I, Tamai S. Arthroscopic drilling for the treatment of osteochondral lesions of the talus. J Bone Joint Surg Am. 1999; 81:1229-1235.

12. O'Farrell TA, Costello BG. Osteochondritis dissecans of the talus: the late results of surgical treatment. J Bone Joint Surg Br. 1982; 64:494-497.

13. Scharling M. Osteochondritis dissecans of the talus. Acta Orthop Scand. 1978;49:89-94.

14. Schenck RC Jr, Goodnight JM. Osteochondritis dissecans. J Bone Joint Surg Am. 1996; 78:439-456.

15. Cahill BR. Osteochondritis dissecans of the knee: treatment of juvenile and adult forms. J Am Acad Orthop Surg. 1995; 3:237-247.

16. McCoy AM, Toth F, Dolvik NI, et al. Articular osteochondrosis: a comparison of naturally-occurring human and animal disease. Osteoarthritis Cartilage. 2013;21:1638-47.

17. Blumer MJ, Longato S, Richter E, Pérez MT, Konakci KZ, Fritsch $\mathrm{H}$. The role of cartilage canals in endochondral and perichondral bone formation: are there similarities between these two processes? J Anat. 2005;206:359-372.

18. Ytrehus B, Ekman S, Carlson CS, Teige J, Reinholt FP. Focal changes in blood supply during normal epiphyseal growth are central in the pathogenesis of osteochondrosis in pigs. Bone. 2004; 35:1294-1306.

19. Olstad K, Ytrehus B, Ekman S, Carlson CS, Dolvik NI. Epiphyseal cartilage canal blood supply to the tarsus of foals and relationship to osteochondrosis. Equine Vet J. 2008;40:30-39.

20. Carlson CS, Cullins LD, Meuten DJ. Osteochondrosis of the articular-epiphyseal cartilage complex in young horses: evidence for a defect in cartilage canal blood supply. Vet Pathol. 1995;32:641-647.

21. Baker CL 3rd, Romeo AA, Baker CL Jr. Osteochondritis dis- secans of the capitellum. Am J Sports Med. 2010; 38:19171928.

22. Hixon AL, Gibbs LM. Osteochondritis dissecans: a diagnosis not to miss. Am Fam Physician. 2000; 61:151-158.

23. Novotny H. Osteochondrosis dissecans in two brothers; the pre- and developed state. Acta Radiol. 1952;37:493-497.

24. Pick MP. Familial osteochondritis dissecans. J Bone Joint Surg Br. 1955;37-B:142-145.

25. Hammett RB, Saxby TS. Osteochondral lesion of the talus in homozygous twins-the question of heredity. Foot Ankle Surg. 2010;16:e55-56.

26. Mubarak SJ, Carroll NC. Familial osteochondritis dissecans of the knee. Clin Orthop Relat Res. 1979;140:131-136.

27. Phillips HO, Grubb SA. Familial multiple osteochondritis dissecans. Report of a kindred. J Bone Joint Surg Am. 1985;67:155-156.

28. Naumetz VA, Schweigel JF. Osteocartilagenous lesions of the talar dome. J Trauma. 1980; 20:924-927.

29. Letts M, Davidson D, Ahmer A. Osteochondritis dissecans of the talus in children. J Pediatr Orthop. 2003; 23:617-625.

30. Higuera J, Laguna R, Peral M, Aranda E, Soleto J. Osteochondritis dissecans of the talus during childhood and adolescence. J Pediatr Orthop. 1998;18:328-332.

31. Canale ST, Belding RH. Osteochondral lesions of the talus. J Bone Joint Surg Am. 1980; 62:97-102.

32. Eskesen B. En sjaeldenintraarticulaer talus-fractur. Nord Med. 1942;16:3436-3438.

33. De Smet AA, Fisher DR, Burnstein MI, Graf BK, Lange RH. Value of MR imaging in staging osteochondral lesions of the talus (osteochondritis dissecans): results in 14 patients. AJR Am J Roentgenol. 1990;154:555-558.

34. Alexander H, Lichtman D. Surgical treatment of transchondraltalar dome fractures (osteochondritis dissecans). J Bone Joint Surg Am. 1980; 62:646-652.

35. Pettine KA, Morrey BF. Osteochondral fractures of the talus. J Bone Joint Surg Br. 1987; 69:82-92.

36. Perumal V, Wall E, Babekir N. Juvenile osteochondritis dissecans of the talus. J Pediatr Orthop. 2007; 27:821-825.

37. Verhagen RA, Struijs PA, Bossuyt PM, van Dijk CN. Systematic review of treatment strategies for osteochondral defects of the talar dome. Foot Ankle Clin. 2003;8:233-242.

38. Zengerink M, Struijs PA, Tol JL, van Dijk CN. Treatment of osteochondral lesions of the talus: a systematic review. Knee Surg Sports Traumatol Arthrosc. 2010;18:238-246.

39. Kono M, Takao M, Naito K. Retrograde drilling for osteochondral lesions of the talar dome. Am J Sports Med. 2006;34:1450-1456.

40. Taranow WS, Bisignani GA, Towers JD. Retrograde drilling of osteochondral lesions of the medial talar dome. Foot Ankle Int. 1999;20:474-480.

41. Robinson DE, Winson IG, Harries WJ. Arthroscopic treatment of osteochondral lesions of the talus. J Bone Joint Surg Br. 2003;85:989-993.

42. Gobbi A, Francisco RA, Lubowitz JH, Allegra F, Canata G. Osteochondral lesions of the talus: randomized controlled trial comparing chondroplasty, microfracture, and osteochondral autograft transplantation. Arthroscopy. 2006;22:10851092.

43. O'Driscoll SW. The healing and regeneration of articular cartilage. J Bone Joint Surg Am. 1998;80: 1795-1812.

44. Chuckpaiwong B, Berkson EM, Theodore GH. Micro-fracture for osteochondral lesions of the ankle: outcome analysis and outcome predictors of 105 cases. Arthroscopy. 2008; 24:106-112.

45. Kouvalchouk JF, Schneider-Maunoury G, Rodineau J. 
Osteochondral lesions of the dome of the talus with partial necrosis. Surgical treatment by curettage and filling. Rev Chir Orthop Reparatrice Appar Mot. 1990;76:480-489.

46. Scranton PE Jr, Frey CC, Feder K. Outcome of osteochondral autograft transplantation for type- $\mathrm{V}$ cystic osteochondral lesions of the talus. J Bone Joint Surg Br. 2006;88:614-619.

47. Hangody L, Fules P. Autologous osteochondral mosaicplasty for the treatment of full-thickness defects of weight-bearing joints: ten years of experimental and clinical experience. J Bone Joint Surg Am. 2003;2:25-32.

48. Giannini S, Battaglia M, Buda R, Cavallo M, Ruffilli A, Vannini F. Surgical treatment of osteochondral lesions of the talus by open-field autologous chondrocyte implantation: a 10 -year follow-up clinical and magnetic resonance imaging T2-mapping evaluation. Am J Sports Med. 2009;37 Suppl 1:112S-118S.

49. Valderrabano V, Miska M, Leumann A, Wiewiorski M. Reconstruction of osteochondral lesions of the talus with autologous spongiosa grafts and autologous matrix-induced chondrogenesis. Am J Sports Med. 2013;41:519-527.

50. Zwingmann J, Südkamp NP, Schmal H, Niemeyer P. Surgical treatment of osteochondritis dissecans of the talus: a systematic review. Arch Orthop Trauma Surg. 2012;132:1241-1250. 